\title{
Формально-кинетический подход к описанию кинетики гидратации алюмосиликатов
}

\author{
(C) 2021 Котова Д.Л. ${ }^{1}$, Крысанова Т.А. ${ }^{1}$, Новикова Л.А. ${ }^{2}$, Крысанов В.А. ${ }^{1}$ \\ ${ }^{I}$ Воронежский государственный университет, Воронеж \\ ${ }^{2}$ Воронежский государственный лесотехнический университет им. Г.Ф. Морозова, Воронеж
}

Поступила в редакцию 26.01.2021 г.

DOI: $10.17308 /$ sorpchrom.2021.21/3351

Учет особенностей транспорта воды как основного компонента системы важен при исследовании кинетических характеристик сорбционного процесса с участием природных алюмосиликатов, которые находят широкое применение в качестве сорбентов активных и вспомогательных веществ фармацевтической продукции. При изучении кинетических закономерностей адсорбции воды алюмосиликатами представляет интерес решение задачи количественного моделирования процесса.

В качестве объектов исследовании выбраны алюмосиликаты: клиноптилолит Словацкого месторождения и глауконит месторождения юго-запада Воронежской антеклизы, отличающиеся кристаллическим типом структуры и обладающие способностью к ионному обмену. Кинетику адсорбции воды алюмосиликатами изучали методом изопиестирования при активности воды в паре, равной 0.980 , и температуре $295 \pm 2$ К. Количество адсорбированной воды определяли методом гравиметрии. Кинетическое исследование процесса гидратации алюмосиликатов включало в себя экспериментальное определение зависимости количества адсорбированной воды от времени и математическую обработку данных с использованием кинетических уравнений, основанных на конкретных моделях твердофазного взаимодействия.

Выявлено, что более высокая гидратационная способность (примерно в 1.4 раза) и увеличение скорости установления равновесия в системе алюмосиликат - молекулы воды для клиноптилолита обусловлены большей концентрацией обменных катионов и более развитой пористостью и поверхностью по сравнению со слабо набухающим глауконитом. Выбор функции $g(\alpha)$, соответствующей механизму на каждом этапе процесса гидратации, осуществляли способом проверки линейности зависимости $\lg \mathrm{g}(\alpha)=\tau$ для рассматриваемых уравнений, описывающих механизм адсорбции воды $(\alpha-$ степень достижения равновесия). Из уравнений выбирали такое, которое в заданной области $\alpha$ отвечало минимальному значению дисперсии. Для каждой стадии, лимитирующей процесс в определенном интервале $\alpha$, оценивали кинетические характеристики, используя зависимость $\lg g(\alpha)$ от $\tau$.

Установлено, что на начальном этапе гидратацию алюмосиликатов контролирует химическая стадия. Скорость процесса определяется формированием центров гидратации. На данном этапе клиноптилолит адсорбирует $57 \%$, а глауконит $-51 \%$ от общего количества поглощенной воды. Адсорбцию воды на втором этапе процесса описывает уравнение реакции на межфазной границе. Для глауконита характерно заметное снижение скорости гидратации на данном этапе, что проявляется в значительном уменьшении его гидратационной способности. На конечном этапе гидратации проявляются диффузионные ограничения, процесс описывается уравнением трехмерной диффузии, что отмечается снижением общей скорости адсорбции. Для клиноптилолита характерно более позднее проявление диффузионных ограничений, что обусловлено его структурными характеристиками.

Ключевые слова: алюмосиликаты, кинетика гидратации.

\section{Введение}

В настоящее время природные алюмосиликаты находят широкое прак- тическое применение в качестве эффективных сорбентов, катализаторов, активных и вспомогательных веществ фармацевтической продукции [1-4]. Закономерности транспорта воды, как ос- 
новного компонента сорбционной системы, в значительной степени определяют кинетические характеристики сорбционного процесса с участием алюмосиликатов. Гидратационная способность алюмосиликатов определяется их кристаллохимической структурой, текстурными характеристиками, природой и количеством обменных катионов [5-8]. В природных алюмосиликатах, называемых также водными алюмосиликатами, вода находится не только в адсорбированном состоянии, но также является основным элементом кристаллической структуры минералов (конституционная вода) в виде гидроксильных групп различного характера (мостиковых, силанольных, алюминольных). В связи с этим, структура алюмосиликатов очень восприимчива к таким параметрам, как давление паров воды, температура и др. факторов, и может существенно меняться при изменениивнешнего окружения минералов $[6,9,10]$. При исследовании кинетических закономерностей адсорбции воды алюмосиликатами возникает задача количественного моделирования процесса. Взаимодействие молекул воды с алюмосиликатами с кинетической точки зрения можно представить как простую гетерогенную реакцию.

В данной работе приведены результаты исследования кинетической закономерности адсорбции воды алюмосиликатами, различающимися кристаллическим типом структуры и физико-химическими свойствами.

\section{Экспериментальная часть}

В качестве объектов исследовании выбраны алюмосиликаты клиноптилолит Словацкого месторождения (Клн) и глауконит месторождения юго-запада Воронежской антеклизы (Глт), отличающиеся кристаллическим типом структуры и обладающие выраженной способностью к ионному обмену. Клиноптилолит является представителем каркасных алюмосиликатов с мольным отношением $\mathrm{Si} / \mathrm{Al}$ в его структуре равным 5,5. Отрицательный заряд каркаса матрицы $\left(\mathrm{AlO}_{4}\right)^{-}$и компенсирующие его положительно заряженные внекаркасные катионы $\left(\mathrm{K}^{+}\right.$, $\mathrm{Ca}^{2+}$ и $\left.\mathrm{Na}^{+}\right)$определяют локальное распределение заряда на поверхности полостей и каналов, доступных для молекул воды. Суммарный объем пор для клиноптилолита равен $0.44 \mathrm{~cm}^{3} / \Gamma$, средний диаметр пор - 11.05 нм [11,12].

Глауконит - природный минерал слоистой структуры ряда иллита. Выбранный для исследования образец глауконита представлен смешанно-слойными минералами ряда иллит-смектит (I : S) в соотношении 50:50 и 50:80. В межслоевом пространстве присутствуют обменные катионы $\left(\mathrm{K}^{+}, \mathrm{Mg}^{2+}\right.$ и $\left.\mathrm{Ca}^{2+}\right)$ и молекулы воды. Для глауконита суммарный объем пор и средний диаметр пор составляют соответственно $0.31 \mathrm{~cm}^{3} / \Gamma$ и 5.7 нм [13].

Кинетику адсорбции воды алюмосиликатами изучали методом изопиестирования [14]при активности воды в паре $\left(a_{w}\right)$, равной 0.980 и температуре $295 \pm 2$ К. Образцы алюмосиликатов предварительно дегидратированные при 473 К (Клн) и 423 К (Глт), затем выдержанные над $\mathrm{P}_{2} \mathrm{O}_{5}$ до постоянной массы [15], приводили в изопиестическое равновесие с насыщенным раствором $\mathrm{K}_{2} \mathrm{Cr}_{2} \mathrm{O}_{7}$. Количество адсорбированной воды определяли методом гравиметрии. Образцы сорбентов периодически взвешивали в течение всего времени установления равновесия в системе. По изменению массы алюмосиликатов рассчитывали степень достижения равновесия $(\alpha)$ в момент времени $(\tau): \alpha=\mathrm{n}_{\tau} / \mathrm{n}_{\infty}$, где $\mathrm{n}_{\tau}-$ количество адсорбированной воды в данный момент времени $\tau$ (ммоль/г), $\mathrm{n}_{\infty}-$ количество адсорбированной воды при достижении равновесия в исследуемой системе вода - алюмосиликат (ммоль/г).

\section{Обсуждение результатов}

При рассмотрении кинетических закономерностей гидратации алюмосиликатов принимали во внимание результа- 
ты исследования равновесной адсорбции воды [5]. Изотермы адсорбции воды на клиноптилолите и глауконите имеют S-образный вид, связанный со ступенчатым заполнением межпакетного пространства сорбентов. В области низкой активности растворителя молекулы воды локализуются у активных центров, в качестве которых выступают внекаркасные катионы и электроотрицательные центры каркаса сорбента, образуя монослой. С повышением активности воды адсорбция протекает в результате образования ассоциатов воды. При активности воды выше 0.700 существенное значение приобретает капиллярная конденсация в мезопорах сорбентов, присутствие которых обусловлено вторичной пористостью $[16,17]$. Показано, что различие в гидратационной способности каркасного и слоистого алюмосиликатов во всей области активности воды обусловлено природой и количеством обменных катионов, величиной свободного объема и размера полостей в структуре сорбентов.

Кинетическое исследование процесса гидратации алюмосиликатов включало два этапа: экспериментальное определение зависимости количества адсорбированной воды от времени (рис.1, а, б) и математическая обработка полученных кинетических данных с использованием кинетических уравнений, основанных на конкретных моделях твердофазного взаимодействия [18].

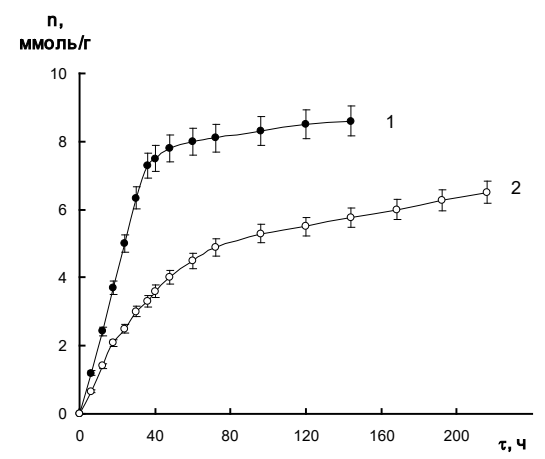

a
Природа активных центров, их плотность распределения на поверхности сорбента оказывают влияние на ход кинетической кривой, время достижения равновесия и количество максимально поглощенного растворителя. Начальный участок кинетических кривых в течение 48 часов для Клн и 24 часов Глт характеризуется более высокой скоростью поглощения воды сорбентами. В дальнейшем процесс гидратации алюмосиликатов замедляется и приводит к установлению равновесия для Клн через 144 , а для Глт - 216 часов. Количество адсорбированной воды в состоянии равновесия для Клн и Глт составило соответственно 8.6 и 6.5 ммоль/г.

Более высокая гидратационная способность (примерно в 1.4 раза) и увеличение скорости установления равновесия в системе алюмосиликат - молекулы воды для Клн обусловлены большей концентрацией обменных катионов и более развитой пористостью и поверхностью по сравнению со слабо набухающим Глк [11-13].

Наблюдаемые различия в скорости адсорбции воды, независимо от ее количества, подтверждают существование в алюмосиликатах определенной структуры воды из сетки водородных связей [19, 20]. Экспериментальные данные, представленные в координатах $-\lg \alpha-\tau$ (рис. 2) указывают на различную скорость гидратации в определенном интер-

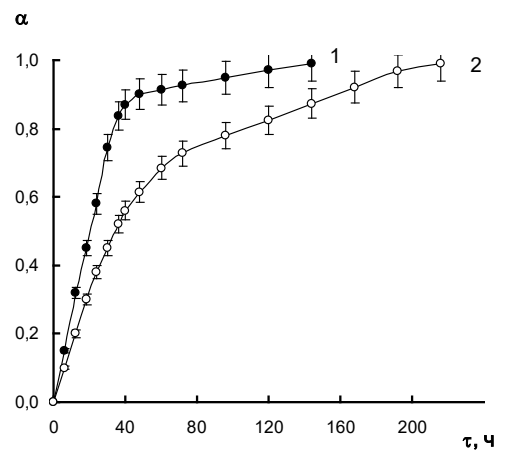

б

Рис.1. Кинетические кривые адсорбции воды клиноптилолитом (1) и глауконитом (2) в координатах: a) $\mathrm{n}-\tau$; б) $\alpha-\tau$.

Fig. 1. Kinetic curves of water adsorption by clinoptilolite (1) and glauconite (2) in the coordinates: a) $n-\tau$; b) $\alpha-\tau$. 


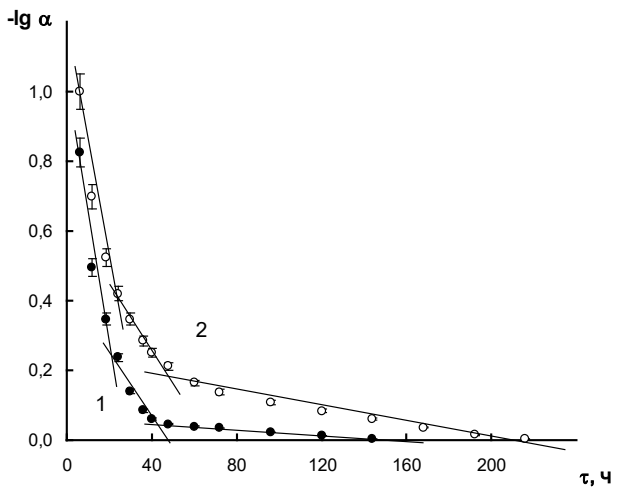

Рис.2 Кинетические кривые адсорбции воды клиноптилолитом (1) и глауконитом (2) в координатах $-\lg \alpha-\tau$

Fig. 2 Kinetic curves of water adsorption by clinoptilolite (1) and glauconite (2) in the coordinates $-\log \alpha-\tau$

вале $\alpha$ и предполагают три кинетически неравноценные формы связи воды.

Построение макрокинетической модели адсорбции воды с учетом стадийности процесса проводили, используя кинетические уравнения, основанные на конкретных моделях твердофазного взаимодействия [18]. В качестве лимитирующей стадии (определяющей скорость процесса на данном этапе) рассматривали следующие процессы: а) объемную диффузию молекул воды через слой продуктов реакции; б) взаимодействие молекул воды с активными центрами в реакционной зоне на границе раздела фаз; в) образование и рост зародышей новой фазы, являющейся продуктом реакции.

Выбор функции $\mathrm{g}(\alpha)$, соответствующей механизму на каждом этапе процесса гидратации, осуществляли способом проверки линейности зависимости $\operatorname{lgg}(\alpha)=\tau$ для рассматриваемых теоретически обоснованных уравнений, описывающих механизм адсорбции воды [21]. Скорость процесса гидратации в определенном интервале степени превращения $\alpha$ может определяться взаимодействием молекул воды с активными центрами в структуре алюмосиликатов, с образованием новых реакционных центров, а также может контролироваться либо реакцией на межфазной границе, либо диффузией молекул воды через слой продукта к активным центрам. Из уравнений, отвечающих определенным кинетическим моделям, выбирали такое, которое в заданной области $\alpha$ отвечало минимальному значению дисперсии (Д), то есть описывало гидратацию на данном этапе с максимальной вероятностью. Для каждой стадии, лимитирующей процесс в определенном интервале $\alpha$, оценивали кинетические характеристики, используя

Таблица. Кинетические характеристики адсорбции воды алюмосиликатами $\left(a_{w}=0.980\right)$

Table. The kinetic characteristics of water adsorption by aluminosilicates $\left(a_{w}=0.980\right)$

\begin{tabular}{|c|c|c|c|c|c|c|}
\hline Сорбент & Интервал $\alpha$ & $\begin{array}{c}\text { Вид функции } \\
\mathrm{g}(\alpha)\end{array}$ & $\begin{array}{c}\mathrm{n}, \\
\text { ммоль/г; } \\
(\%)\end{array}$ & $\begin{array}{c}\text { Коэффи- } \\
\text { циент } \\
\text { диффузии } \\
\mathrm{D} \cdot 10^{7}, \\
\mathrm{~cm}^{2} / \mathrm{c}\end{array}$ & $\begin{array}{c}\text { Констан- } \\
\text { та } \\
\text { скорости } \\
\mathrm{k} \cdot 10^{2}, \\
\mathrm{c}^{-1}\end{array}$ & $\begin{array}{c}\text { Диспер- } \\
\text { сия } \\
\text { Д·10 }\end{array}$ \\
\hline Клино- & $0.10-0.57$ & $-\lg (1-\alpha)^{1 / 3}$ & $4.9(57.0)$ & - & 10.2 & 0.09 \\
птилолит & $0.57-0.91$ & $1-(1-\alpha)^{1 / 3}$ & $2.8(32.5)$ & - & 5.1 & 2.33 \\
\hline Глауко- & $0.91-0.99$ & {$\left[1-(1-\alpha)^{1 / 3}\right]^{2}$} & $0.9(10.5)$ & 7.11 & - & 2.87 \\
нит & $0.09-0.38$ & $-\lg (1-\alpha)^{1 / 3}$ & $3.3(50.8)$ & - & 7.4 & 0.12 \\
& $0.38-0.65$ & $1-(1-\alpha)^{1 / 3}$ & $1.0(15.4)$ & - & 4.2 & 2.89 \\
{$\left[1-(1-\alpha)^{1 / 3}\right]^{2}$} & $2.2(33.8)$ & 5.82 & - & 3.12 \\
\hline
\end{tabular}


зависимость $\operatorname{lgg}(\alpha)$ от $\tau$.

В таблице приведены количественные характеристики кинетически неравноценных молекул воды, адсорбированных алюмосиликатами при $a_{w}=0.980$, уравнения, контролирующие процесс на данном этапе, значения кинетических параметров и дисперсия (Д) в области линейности функции $\mathrm{g}(\alpha)$.

На начальном этапе гидратацию алюмосиликатов контролирует химическая стадия. Процесс описывается уравнением Авраами-Ерофеева [18]:

$$
-\lg (1-\alpha)^{1 / n}=k \tau \text {, }
$$

где $\mathrm{n}$ - параметр определяемый механизмом реакции, скоростью зародышеобразования и геометрией зародышей. Кинетическое уравнение, для которого область линейности сопровождается минимальной дисперсией, имеет вид:

$$
\mathrm{g}(\alpha)=\lg (1-\alpha)^{1 / 3} \text {. }
$$

Молекулы воды координируются у активных центров алюмосиликата в результате ион-дипольного и дипольдипольного взаимодействий. Скорость процесса определяется формированием центров гидратации. По мере их роста скорость возрастает. Согласно полученным данным на начальном этапе процесса клиноптилолит адсорбирует 57\%, глауконит - $51 \%$ от общего количества поглощенной воды.

Адсорбцию воды на втором этапе процесса описывает уравнение реакции на межфазной границе:

$$
g(\alpha)=1-(1-\alpha)^{1 / 3} .
$$

Скорость процесса определяется величиной доступной межфазной поверхности, т.е. диффузия молекул воды через слой адсорбента происходит достаточно быстро [18,21]. Для глауконита характерно заметное снижение скорости гидратации на данном этапе, что проявляется в значительном уменьшении его гидратационной способности.
На конечном этапе гидратации проявляются диффузионные ограничения. Процесс описывается уравнением трехмерной диффузии:

$$
\mathrm{g}(\alpha)=\left[1-(1-\alpha)^{1 / 3}\right]^{2} .
$$

Особенность протекания процесса в условиях лимитирования диффузией отмечается в снижении общей скорости адсорбции. Для клиноптилолита характерно более позднее проявление диффузионных ограничений, что обусловлено структурными характеристиками сорбента [11-13].

\section{Заключение}

Таким образом, различие в природе и количестве обменных катионов, пористости и поверхности клиноптилолита и глауконита влияют на ход кинетической кривой, время достижения равновесия в системе, гидратационную способность алюмосиликатов и кинетические характеристики отдельных стадий адсорбционного процесса. Более высокие гидратационная способность и скорость установления равновесия в системе «алюмосиликат - молекулы воды» для клиноптилолита связаны с большей концентрацией обменных катионов и более развитой пористостью и поверхностью по сравнению со слабо набухающим глауконитом. При рассмотрении макрокинетической модели адсорбции воды установлено, что на начальном этапе гидратацию алюмосиликатов контролирует химическая стадия, второй этап гидратации описывается уравнением реакции на межфазной границе, конечный этап уравнением трехмерной диффузии. Для клиноптилолита характерно более позднее проявление диффузионных ограничений, что обусловлено его структурными характеристиками. 


\section{Список литературы}

1. Boudissa F., Mirilà D., Arus A-V., Thizizi Terkmani et al. // J. Hazard Mater. 2019. Vol. 364. pp. 356-366.

2. Cerri Guido, Farina M., Brundu A., Daković A. et al. // Microporous and Mesoporous Materials. 2016. Vol. 223. pp. 5867.

3. Novikova L., Belchinskaya L. // Clays, Clay Minerals and Ceramic Materials Based on Clay Minerals. 2016. pp. 89-128.

4. Kotova D.L., Vasilyeva S.Y., Krysanova T.A., Khromova A.S. et al. // Protection of Metals and Physical Chemistry of Surfaces. 2015. Vol. 51. No4. pp .499-504.

5. Kotova D.L., Artamonova M.N., Krysanova T.A. Vasilenko M.S. et al. // Protection of Metals and Physical Chemistry of Surfaces. 2018. Vol. 54. No 4. pp. 598-602.

6. Korkuna O., Leboda R., SkubiszewskaZieba J. // Microporous Mesoporous Mater. 2006. Vol. 87. pp. 243-254.

7. Брек Д. Цеолитовые молекулярные сита. М. Мир. 1976.760 с.

8. White D.A., Bussey R.L. // Separ. Purif. Technol. 1997. Vol. 11. pp. 137-141.

9. Alver B.E., Sakizci M., Yorukogullari E. // J. Therm. Anal. Calorim. 2010. Vol. 100. Iss. 1. pp. 19-26.

10. Arcoya A., Gonzalez J., Llabre G., Seoane X.L. et al. // Microporous Mesoporous Mater. 1996. Vol. 7. pp. 1-13.

11. Бельчинская Л.И., Ходосова Н.А., Битюцкая Л.А // Физикохимия поверхности и защчита материалов. 2009. Т. 45. № 2. С. 218-221.

12. Бельчинская Л.И., Ходосова Н.А., Стрельникова О.Ю., Петухова Г.А. и др. // Физикохимия поверхности и защита материалов. 2015. Т. 51. № 5. С. 1-8.

13. Novikova L, Belchinskaya L, Krupskaya V., Roessner F. et al. // Сорбционные и хроматографические процессы. 2015. Т. 15. № 5. C. $730-740$.

14. Киргинцев А.Н. Очерки о термодинамике водно-солевых растворов. Новосибирск: Наука. 1976. 201 с.

15. Черенкова Ю.А., Котова Д.Л., Селеменев В.Ф., Крысанова Т.А. и др. // Журн. физ. химии. 2005. Т. 79. № 4. С. 716-720.

16. Kowalczyk P., Myroslav S., Artur P.T. // Journal of Colloid and Interface Science. 2006. Vol. 297. No 1. pp. 77-85.

17. Sprynskyy M., Golembiewski R., Trykowski G., Buszewski B. // Journal of Physicsand Chemistry of Solids. 2010. Vol. 71. No 9. pp. 1269-1277.

18. Третьяков Ю.Д. Твердофазные реакции. М. Химия. 1978. 172 с.

19. Channon Y.M., Catlow C.R.A., Jackson R.A. // Studies in Surface Science and Catalysis. 1995. Vol. 97. pp. 117-124.

20. Borowski M., Wolf I., Gies H. // Chem. Mater. 2002. No 14(1). pp. 38-43.

21. Дельмон Б. Кинетика гетерогенных реакций. М. Мир. 1972. 544 с.

\title{
Formal kinetic approach for the description of the kinetics of hydration of aluminosilicates
}

\author{
(C) 2021 Kotova D.L. ${ }^{1}$, Krysanova T.A. ${ }^{1}$, Novikova L.A. ${ }^{2}$, Krysanov V.A. ${ }^{1}$ \\ ${ }^{1}$ Voronezh State University, Voronezh, Russian Federation \\ ${ }^{2}$ Voronezh State University of Forestry and Technologies Named after G.F. Morozov,Voronezh, Russian \\ Federation
}

The consideration of the peculiarities of water transport as the main component of a system is important when studying the kinetic characteristics of the sorption process with the participation of natural aluminosilicates, which are widely used as sorbents for active and auxiliary substances in pharmaceutical products. When studying the kinetic regularities of water adsorption by aluminosilicates, the problem of quantitative modelling of the process is of interest.

The objects of study were aluminosilicates: clinoptilolite from the Slovatsk deposit and glauconite from the south-west of the Voronezh anteclise, which are characterized by a crystalline structure and have the ability to perform ion exchange. The kinetics of water adsorption by aluminosilicates was studied by isopiesisation with water activity in vapour equal to 0.980 and a temperature of $295 \pm 2 \mathrm{~K}$. The amount of adsorbed 
water was determined by gravimetry. The kinetic study of the process of hydration of aluminosilicates included the experimental determination of the dependence of the amount of adsorbed water on time and mathematical processing of the data using kinetic equations based on specific models of solid-phase interaction.

It was revealed that a higher hydration capacity (by about 1.4 times) and an increase in the rate of reaching the equilibrium in the system of aluminosilicate - water molecules for clinoptilolite were due to a higher concentration of exchangeable cations and a more developed porosity and surface compared to weakly swelling glauconite.

The choice of the function $\mathrm{g}(\alpha)$ corresponding to the mechanism at each stage of the hydration process was carried out by checking the linearity of the dependence $\log g(\alpha)=\tau$ for the considered equations describing the mechanism of water adsorption ( $\alpha$ - the degree of reaching the equilibrium). From the equations, we chose the one that, in the given region $\alpha$, corresponded to the minimum value of the dispersion. For each stage, limiting the process in a certain range of $\alpha$, the kinetic characteristics were estimated using the dependence of $\log g(\alpha)$ on $\tau$.

It was found that at the initial stage, the hydration of aluminosilicates was controlled by the chemical stage. The rate of the process was determined by the formation of hydration centres. During this stage, clinoptilolite adsorbed $57 \%$, and glauconite adsorbed $51 \%$ of the total amount of absorbed water. The adsorption of water during the second stage of the process was described by the reaction equation at the interface. Glauconite was characterized by a noticeable decrease in the rate of hydration during this stage, which was manifested in a significant decrease in its hydration capacity. During the final stage of hydration, diffusion restrictions appeared and the process was described by the equation of three-dimensional diffusion and it was characterized by a decrease in the overall rate of adsorption. Clinoptilolite was characterized by a later manifestation of diffusion restrictions, which was due to its structural characteristics.

Keywords: aluminosilicates, hydration kinetics

\section{References}

1. Boudissa F., Mirilà D., Arus A-V., Thizizi Terkmani et al., J. Hazard Mater., 2019, Vol. 364, pp. 356-366. DOI :10.1016/j.jhazmat. 2018.09.070

2. Cerri Guido, Farina M., Brundu A., Daković A. et al., Microporous and Mesoporous Materials, 2016, Vol. 223, pp. 58-67. DOI: 10. 1016/j.micromeso.2015.10.034

3. Novikova L., Belchinskaya L., Clays, Clay Minerals and Ceramic Materials Based on Clay Minerals, 2016, pp. 89-128. DOI: 10.5772/61678

4. Kotova D.L., Vasilyeva S.Y., Krysanova T.A., Khromova A.S. et al., Protection of Metals and Physical Chemistry of Surfaces, 2015, Vol. 51, No 4, pp. 499-504. DOI: 10.1134/ S207020511504019X

5. Kotova D.L., Artamonova M.N., Krysanova T.A. Vasilenko M.S. et al., Protection of Metals and Physical Chemistry of Surfaces, 2018, Vol. 54, No 4, pp. 598-602. DOI: 10.1134/ S2070205118030073

6. Korkuna O., Leboda R., SkubiszewskaZieba J., Microporous Mesoporous Mater. 2006, Vol. 87, pp. 243-254. DOI: 10.1016/ j.micromeso.2005.08.002

7. Breck D. Zeolitjvye molekulyarnye sita, M., Mir. 1976. $760 \mathrm{p}$.

8. White D.A., Bussey R.L., Separ. Purif. Technol., 1997, Vol. 11, pp. 137-141.
9. Alver B.E., Sakizci M., Yorukogullari E., J. Therm. Anal. Calorim., 2010, Vol. 100, Iss.1, pp. 19-26. DOI: 10.1007/s10973-009-0118-0

10.Arcoya A., Gonzalez J., Llabre G., Seoane X.L. et al., Microporous Mesoporous Mater., 1996, Vol. 7, pp. 1-13. DOI: 10.1016/09276513(96)00022-3

11.Belchinskaya L.I., Khodosova N.A., Bityutskaya L.A., Physicochemistry of Surface and Protection of Materials, 2009, Vol. 45, No 2, pp. 218-221.

12.Belchinskaya L.I., Khodosova N.A., Strelnikova O.Yu., Petuhova G.A. et al., Physicochemistry of surfaces and protection of materials, 2015, Vol. 51, No 5, pp. 1-8. DOI: 10.7868/S0044185615050046

13.Novikova L, Belchinskaya L, Krupskaya V., Roessner F. et al., Sorptsionnye I khromatograficheskie protsessy, 2015, Vol. 15, No 5, pp. 730-740. DOI: $10.17308 /$ sorpchrom. $2015.15 / 327$

14.Kirgintsev A.N. Essays on the thermodynamics of water-salt solutions. Novosibirsk, Nauka, 1976, 201 p.

15.Cherenkova Yu.A., Kotova D.L., Selemenev V.F. et al., Journal of physical chemistry, 2005, Vol. 79, No 4, pp. 716-720.

16.Kowalczyk P., Myroslav S., Artur P.T., Journal of Colloid and Interface Science, 2006, Vol. 297, No 1, pp. 77-85. DOI: 10.1016 / j. jcis.2005.10.045

17.Sprynskyy M., Golembiewski R., Trykowski G., Buszewski B., Journal of Physicsand 
Chemistry of Solids, 2010, Vol. 71, No 9, pp. 1269-1277. DOI:10.1016/j.jpcs.2010.05.006

18.Tretyakov Yu.D., Solid phase reactions, M., Chemistry, 1978, $172 \mathrm{p}$.

19.Channon Y.M., Catlow C.R.A., Jackson R.A., Studies in Surface Science and Catalysis,

Котова Диана Липатьевна - д.Х.н., профессор кафедры аналитической химии, Воронежский государственный университет, Воронеж

Крысанова Татьяна Анатольевна - к.х.н., доцент кафедры аналитической химии, Воронежский государственный университет, Воронеж

Новикова Людмила Анатольевна - к.х.н., доцент кафедры химии, Воронежский государственный лесотехнический университет им. Г.Ф Морозова, Воронеж

Крысанов Вячеслав Александрович - к.Х.н., доцент кафедры физической химии, Воронежский государственный университет, Воронеж
1995, Vol. 97, pp. 117-124. DOI: 10.1016/ S0167-2991(06)81880-X

20.Borowski M., Wolf I., Gies H., Chem. Mater., 2002, No 14(1), pp. 38-43.

21.Delmont B., Kinetics of Heterogeneous Reactions, M ., Mir, 1972, 544 p.

Kotova Diana L. - Professor at the Department of Analytical Chemistry, Voronezh State University, Voronezh

Krysanova Tatiana A. - Ph.D, Associate Professor at the Department of Analytical Chemistry, Voronezh State University, Voronezh, Voronezh, Email : takrys@yandex.ru

Novikova Lyudmila A. - Ph.D, Associate Professor at the Department of Chemistry, Voronezh State University of Forestry and Technologies named after G.F. Morozov, Voronezh, E-mail : yonk@mail.ru

Krysanov Viacheslav A. - Ph.D, Associate Professor at the Department of Physical Chemistry, Voronezh State University, Voronezh, E-mail : krysanov@.chem.vsu.ru 